\title{
Design of Aquatic Quadcopter with Hold Position Control and Gimbal Controller for capturing Underwater Video
}

\author{
Ronny Mardiyanto and Komang Trisma Trisuta \\ Electrical Engineering Department, Institut Teknologi Sepuluh Nopember \\ ronny@elect-eng.its.ac.id
}

\begin{abstract}
The paper presents design of an aquatic quadcopter which have four driving forces enabling it to move upward, downward, forward, and backward for capturing underwater video. The system will be used for taking underwater video that usually can be performed by the diver. The current issue in aquatic quadcopter researchs is how the system can automatically maintain its roll, pitch, and depth position for producing smooth video. The existing systems still adopted the manual-mode control that only can operated by the expert operator. The proposal designs and realizes an aquatic quadcopter system having features such as hold position control and camera stabilization for capturing smooth underwater video. The system utilizes IMU sensor MPU 6050 and pressure sensor MS 5803 for measuring the position of the system (roll, pitch, yaw, and depth). The PID controller is used for maintaining the level as well as the depth of aquatic quadcopter. The Arduino Mega is used as main controller as well as sender of telemetry data to ground station. The experiment result shows that the system able to maintain the position automatically the average error of pitch is $1.53 \%$, average error of roll is $1.37 \%$, and average error of depth is $1 \mathrm{Cm}$. The realized system can be used for capturing the underwater video.
\end{abstract}

Keywords: Aquatic quadcopter, Depth Hold, Underwater Videography, Remotely Underwater Operated Vehicle

\section{Introduction}

Nowdays, the development of robot technology has been rising very rapidly. The robot has been succesfully implemented in many areas, one of them is unmanned vehicle. In more general, the robot has been popular to help human activities such as unmanned aerial vehicle for capturing aerial map, unmanned ground vehicle for disposal dangerous object, and underwater remotely operated vehicle for capturing underwater video.

The paper discuss underwater ROV for capturing underwater video. The application of under ROV has been used in many purposes and field. The underwater ROV has been widely used for oil exploration, pipe installation, internet cable inspection, search and rescue application, etc.

Nowdays, the use of underwater ROV for underwater works has more popular due to the economic reason and safety. The risk of involving human for underwater works is high due to many reason such as unindentity dangerous object in underwater circumstance, maximum depth for safe diving, license for diver, etc. It makes the cost for underwater works expensive (The cost for underwater works for one diver can reach USD 300 per hour).

In 2014, there was plane crash (Air Asia QZ8501) in Java Sea. In order to search the Black Box, a lot of personels have been involved due to Java Sea has $320.000 \mathrm{Km}^{2}$. One

Received (November 31, 2017), Review Result (February 6, 2018), Accepted (February 14, 2018) 
of tim that involved to this mission is Baruna Jaya Ship (BJ 1). The ship equipped with underwater ROV showing seabed.

The underwater ROV also has been widely used in the application of marine, military and search and rescue. Especially for search and rescue, It has been recorded that the number of the water accident happened in Indonesia since 2015 is seven accident. All of them involved divers to search and rescue the victims. The search and rescue activities are frequently disturbed by the weather, the ability of divers when on the seabed, temperature of sea water, and time duration.

In marine exploration, monitoring of underwater conditions in general is also done by involving divers. This method relies on the expertise of divers in taking video under the sea and other conditions. Problems will occur if the skill and physical condition of the diver are less than optimal, the safety of the diver will be threatened and the observation will not be maximal.

In this paper, we design the Aquatic Quadcopter equipped with the features of holding positions (roll, pitch, and depth) enabling it to take underwater video smoothly. The system utilizes IMU sensor MPU 6050 and pressure sensor MS 5803 for measuring the position of the system (roll, pitch, yaw, and depth). The PID controller is used for maintaining the level as well as the depth of aquatic quadcopter. The Arduino Mega is used as the main controller as well as the sender of telemetry data to ground station. The designed Aquatic Quadcopter will replace the divers function.

\section{Related Works}

Remotely Operated Vehicle has been developed by many researchers. Ref [2] developed a system for supporting the observation of underwater archaelogy. The system implemented the SURF object detection method for detecting the archaeological objects. The recorded underwater video captured by ROV was used to investigate the underwater archeological site. The difference between the system and the propose system is the absence of a device for maintaing the camera position remains stable.

Ref [3] designed and developed a small Remotely Operated Underwater Vehicle (ROV) with the aim of exploring underwater as a substitute for humans to explore underwater areas that are difficult to be reach and dangerous. The ROV is equipped with powerful underwater probe sensors to collect underwater data, DC brushed motors for directional control and real time camera monitoring. The ROV featured with IMU sensor as a tilt control system to achieve automatic pitch stability and underwater observation via a video camera where the wave control is controlled by using a joystick control mechanism [3]. The difference of the system to the proposed system is the absence of depth control.

Ref [4] conducted a feasibility study to use airborne air propellers. The tests were conducted in the air and water using a test site designed to study the efficiency of rotating multirotor blades. The experimental results showed that the blades can operate well underwater in terms of thrust against power ratios at low rotation speeds without significant cavitation [4]. The system also does not has the depth control.

Ref [5] developed tiny size ROV by adding the camera and underwater TV. It improved the conventional ROV which does not has thrusters. It was used for monitoring the operations in offshore drilling, especially for monitoring the change position of the drill pipe. The system used five pieces of motor, in other side the proposed system uses only four pieces.

Ref [6] conducted stability analysis of underwater ROV which is called DENA. The ROV has six thrusters and the manipulator. Ref [6] improved the automatic control schemes by implemented the PID controller for stabilize the roll, pitch, and depth. The system used six motor drives and the absence of a device that can keep the camera position remains stable. 
Ref [7] designed a ROV that capable for maintaining its position in the desired depth according to the pressure parameters. The system also does not has part to keep the camera position remains stable.

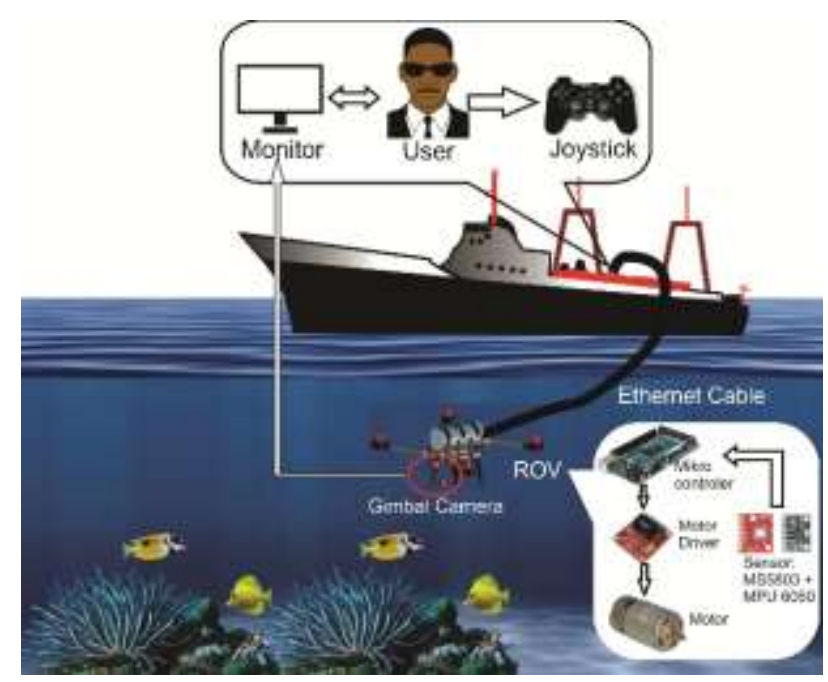

Figure 1. Proposed System

Ref [8] created modelling and linear control of quadcopter. In order stabilize pitch, roll, yaw, and thrust command, they developed derivation of nonlinear mathematical model consisting of six DOF.The PID controller was used to stabilize three DOF rotation. In the proposed system, we propose to be implemented in under water.

Ref [9] conducted an initial performance experiments for underwater cultural heritage survey using ROV driven by six artificial feet with high-resolution acoustic cameras at sea . This ROV used six foot driven by a BLDC motor as its driving force. Therefore, the ROV moved on the seafloor uninterrupted by propellers that are widely used as ROV drives in general. In addition, the robot was expected to minimize the shadow area of the acoustic imaging sensor by controlling its posture correctly using the foot [9]. The system used the legs as a ROV driver.

\section{The Proposed System}

The main objective of the proposed system is capturing underwater video smoothly. The combination between ROV and stabilized camera gimbal can produce smooth underwater video. The captured video is transfered to the FPV (First Person View) display using AV wires. The proposed system utilizes Arduino Mega as main controller, VNH2SP30 as motor drivers, DC motor, and PS2 type of joystick. It uses MPU 6050 sensors as IMU sensor and MS5803 as pressure sensors. The software includes Arduino Mega program using Arduino software and PID control. The PID control is used to control the stability of ROV as well as its depth. The proposed system is powered by 2200 mAh 11.1V 3S. The proposed system is shown in Figure 1. 


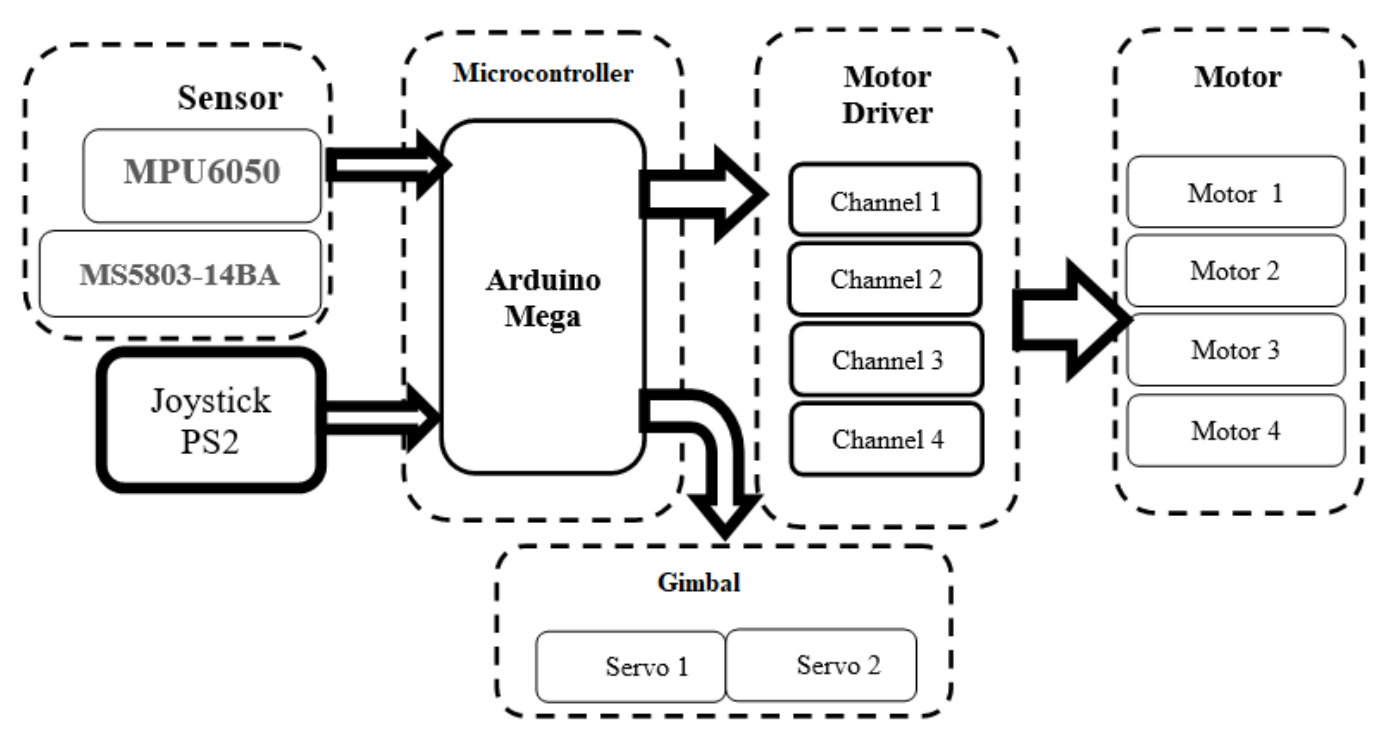

Figure 2. Proposed System

The proposed system uses Arduino Mega type of microcontroller as data processing unit. It uses pressure sensors and IMU sensors for acquiring the position and depth. The pressure sensor is located in the outside of the main tube of ROV for measuring water pressure. The IMU sensors is located in the inside of the main robot tube. The data position that are generated from IMU sensor consist of roll, pitch, and yaw that are used as feedback for stabilizing the ROV. The pressure sensor obtains the water pressure then it is converted to the depth value that is used as feedback for maintaining the depth of ROV. The control method that are used for stabilizing posiiton of ROV and maintaining the depth of ROV is PID controller. A calibration step is performed to take angular values from the IMU when the robot is in flat position which is then stored in the EEPROM. The data stored in EEPROM is used as the PID set point value, so the robot knows the slope. The output of the PID controller is converted to PWM value to set motor rotation as well as the speed. The used pressure sensor uses principal of piezo-resistive to find out the compressive force. The pressure of the ROV start to change if it enters the water, then it will be used to control the depth of the ROV (process variable). The set point is determined using remote control. The process variable and set point are processed by PID controller to obtain the signal control and use it to maintain the depth of ROV. The output of the PID is converted to PWM value to set the speed of motor as well as its rotation. The entire PWM values that are generated from each PID will be summed with the remote control values before proceeding it to the motor via motor driver. The camera gimbal automatically controls its position to always maintain the balance of camera position. The camera gimbal stabilizes its position by using references from IMU sensor (pitch, roll, yaw). The references value can be adjusted from remote control. The data transmission system uses wires by connecting the processing unit located on the robot to the ground station. The block diagram system is shown in Figure 2.

\subsection{Robot Design}

The robot is designed with CAD software, which then some parts are realized using a $3 \mathrm{D}$ printer. The design of proposed system is shown in Figure 3. 


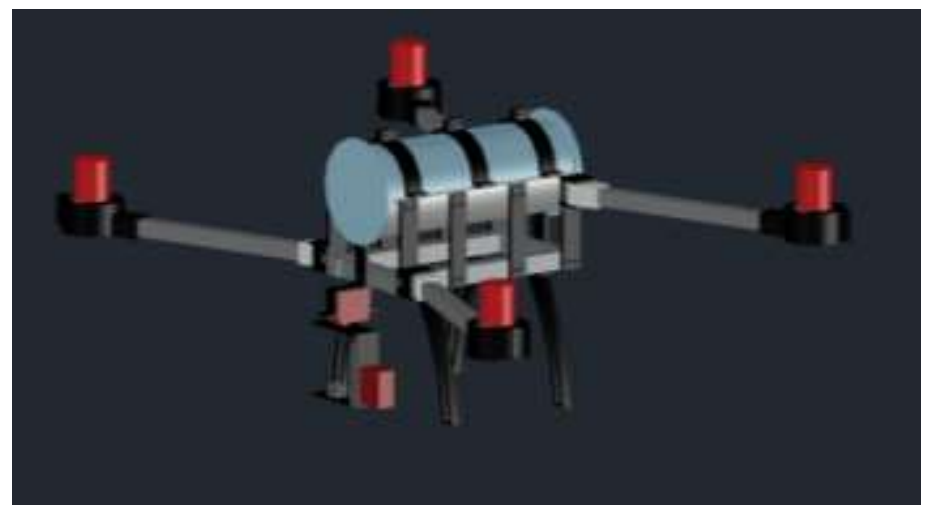

Figure 3. Design of Aquatic Quadcopter

The proposed system uses four motors where each motor has a maximum lift of 1200 gram. The movement of robots can be derived as follows.

The maximum force of each motor is:

$\mathrm{F}=\mathrm{m} \times \mathrm{g}=1200$ gram $\times 10 \mathrm{~m} / \mathrm{s}^{2}=12 \mathrm{~N}$

Where, $\mathrm{F}=$ Lifting Force

$$
\begin{aligned}
& \mathrm{m}=\text { Mass } \\
& \mathrm{g}=\text { Gravity }
\end{aligned}
$$

The calculation of the entire robot by considering the weight of the robot is as follows. Robot mass $(\mathrm{m})=2.47 \mathrm{Kg}$,

Arm Length $(1)=27 \mathrm{Cm}$

Floating Force $\left(\mathrm{F}_{\mathrm{a}}\right)=$ Weight of robot in air - Weight of robot in water

The calculation of the moment of inertia of each axis is as follows.

$$
\begin{aligned}
& I_{x x}=\frac{2 M R^{2}}{5}+2 l^{2} m \\
& I_{y y}=\frac{2 M R^{2}}{5}+2 l^{2} m \\
& I_{z z}=\frac{2 M R^{2}}{5}+4 l^{2} m
\end{aligned}
$$

So,

$$
\begin{aligned}
& \mathrm{I}_{\mathrm{xx}}=\frac{2 \times 2,32 \times(0,05)^{2}}{5}+2 \times(0,27) 2 \times 0,15=0,02419 \\
& \mathrm{I}_{\mathrm{yy}}=\frac{2 \times 2,32 \times(0,05)^{2}}{5}+2 \times(0,27) 2 \times 0,15=0,02419 \\
& \mathrm{I}_{\mathrm{zz}}=\frac{2 \times 2,32 \times(0,05)^{2}}{5}+4 \times(0,27) 2 \times 0,15=0,04606
\end{aligned}
$$

The force acting on the robot is shown in figure 4 . 


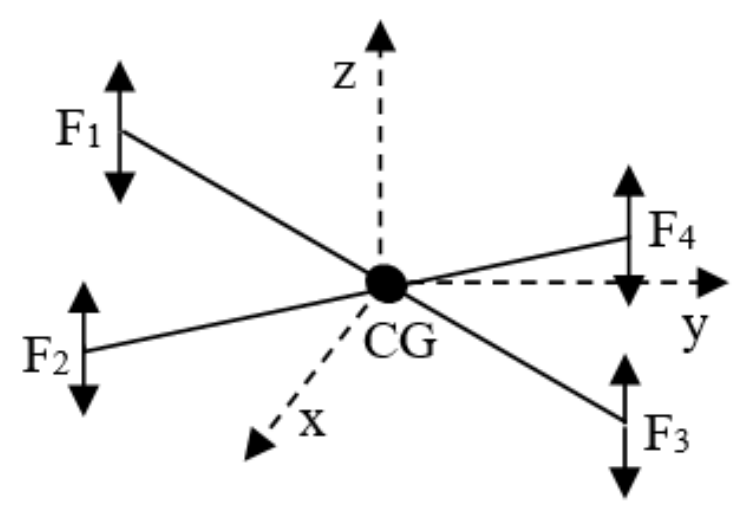

Figure 4. Force Acting on the Robot

From figure 4, then obtained mathematical formulation of robot rotation movement that can be seen in the following calculation.

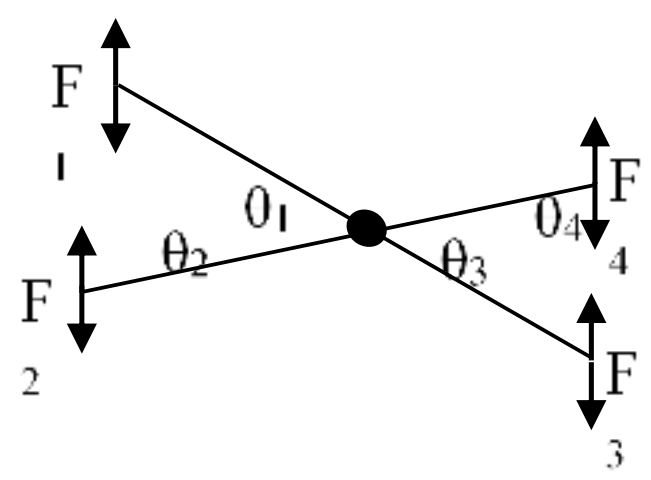

Figure 5 Mathematical Model of Robot Rotation Movement Pitch Axis

$$
\begin{aligned}
& (\mathrm{F} 1+\mathrm{F} 2-\mathrm{F} 3-\mathrm{F} 4) 1 \sin (\theta)=\mathrm{I}_{\mathrm{yy} \cdot} \alpha \\
& \alpha=\frac{(\mathrm{F} 1+\mathrm{F} 2-\mathrm{F} 3-\mathrm{F} 4) \mathrm{l} \sin \theta}{\mathrm{Iyy}}
\end{aligned}
$$

If it is assumed that the motor lift force $(\mathrm{F})$ is equal in each arm, then the result of the calculation is as follows.

$$
\begin{aligned}
& \alpha=\frac{(12+12-12-12) \times 0,27 \times \sin 50^{\circ}}{0,02419} \\
& \alpha=\frac{0 \times 0,27 \times 0,766}{0,02419}=0
\end{aligned}
$$

If $\alpha=0$, then the robot is located in balance condition of pitch (not rotating forward or backward). From the above formulation if the parameter values of the robot are added and the motor lift force is different on each arm, then the $\alpha$ value will be positive or negative. If the value of $\alpha$ is positive then the robot will rotate forward, while if the value of $\alpha$ is negative then the robot will rotate backward based on the orientation that is used to determine the positive or negative value lies in front or back. 


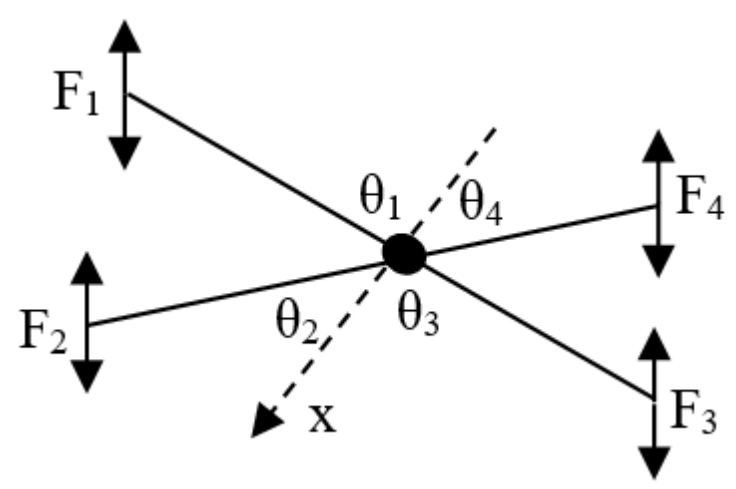

Figure 6. Mathematical Model of Robot Rotation Movement Roll Axis

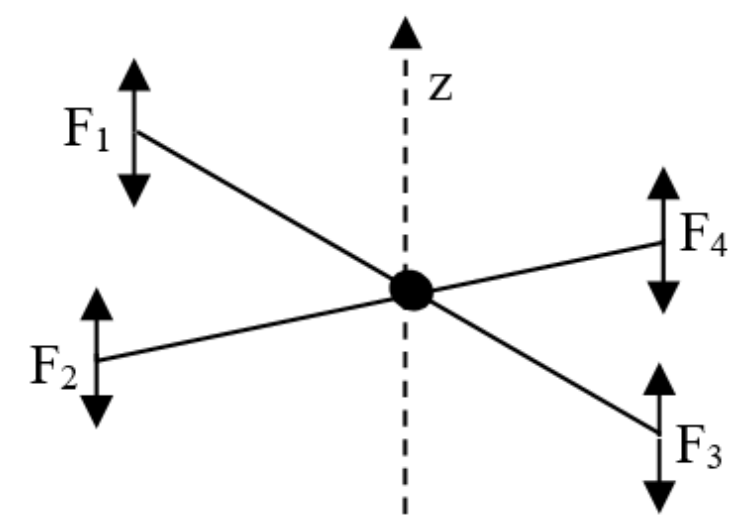

Figure 7. Mathematical Model of Robot Rotation Movement Yaw Axis

$$
\begin{aligned}
& (\mathrm{F} 1+\mathrm{F} 4-\mathrm{F} 2-\mathrm{F} 3) 1 \cos (\theta)=\mathrm{I}_{\mathrm{xx}} \cdot \alpha \\
& \alpha=\frac{(\mathrm{F} 1+\mathrm{F} 4-\mathrm{F} 2-\mathrm{F} 3) \mathrm{I} \cos \theta}{\mathrm{Ixx}}
\end{aligned}
$$

If it is assumed that the motor lift force $(\mathrm{F})$ is equal in each arm, then the result of the calculation is as follows.

$$
\begin{aligned}
& \alpha=\frac{(12+12-12-12) \times 0,27 \times \cos 40^{\circ}}{0,02419} \\
& \alpha=\frac{0 \times 0,27 \times 0,766}{0,02419}=0
\end{aligned}
$$

If $\alpha=0$, then the robot is located in balance condition of roll (not rotating to the right or left). From the above formulation if the parameter values of the robot are added and the motor lift force is different on each arm, then the $\alpha$ value will be positive or negative. If the value of $\alpha$ is positive then the robot will rotate to right, while if the value of $\alpha$ is negative then the robot will rotate left based on the orientation that is used to determine the positive or negative value located on the right or left.

$$
\begin{aligned}
& \left(F_{1}+F_{3}-F_{2}+F_{4}\right) l=I_{z z} \cdot \alpha \\
& \alpha=\frac{(F 1+F 3-F 2+F 4) 1}{I z z}
\end{aligned}
$$




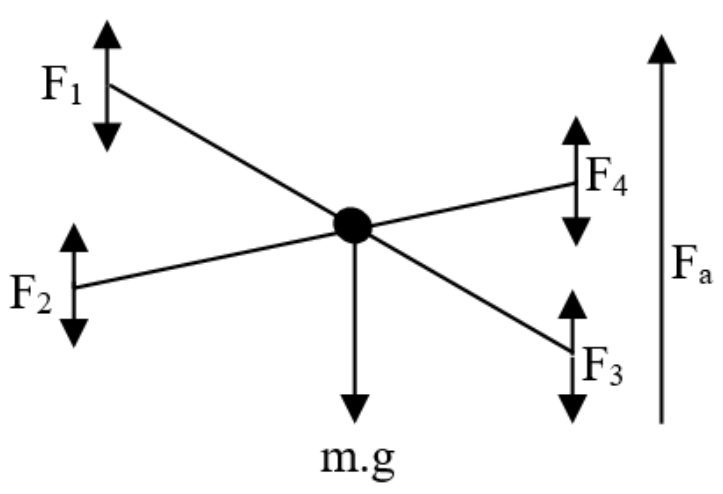

Figure 8. Mathematical Model of Robot Translation Movement

If it is assumed that the motor lift force $(\mathrm{F})$ is equal in each arm, then the result of the calculation is as follows.

$$
\begin{aligned}
& \alpha=\frac{(12+12-12-12) \times 0,27}{0,04606} \\
& \alpha=\frac{0 \times 0,27}{0,046069}=0
\end{aligned}
$$

If $\alpha=0$ then the robot will be in a balanced yaw position does not turn right or left. From the above formulation if the parameter values of the robots are added and the motor lift force is different on each arm, then the $\alpha$ value obtained will be positive or negative value. If the value of $\alpha$ is positive then the robot will turn to the right, while if the value of $\alpha$ is negative then the robot will turn left based on the orientation used to determine the positive or negative value located on the right or left.

From the robot mathematical model in figure 4, we also find mathematical formulation for the translation movement of the robot which can be seen in the following calculations.

$$
\begin{aligned}
& \Sigma F=m a \\
& \left(F_{1}+F_{2}+F_{3}+F_{4}\right)-m g+F_{a}=m a \\
& a=\frac{(F 1+F 2+F 3+F 4)-m \cdot g+F a}{m}
\end{aligned}
$$

From the above formulation if the parameter value of the robot is added, it will get acceleration (a) value is positive or negative. If the value a is positive then the robot will move upwards, while if the value of a is negative then the robot will move down.

\subsection{Camera Gimbal}

The proposed system designs 2 axis camera to for capturing smooth underwater video. It will maintain the roll and pitch positions of camera. The gimbal consist of two motor servos as actuator and arduino as the controller. The motor servos are located in the outside of main tube, in otherside we locate the arduino in the inside of main tube. The PID controller is implemented for maintaining the stability of camera gimbal, it is embeeded in arduino. 


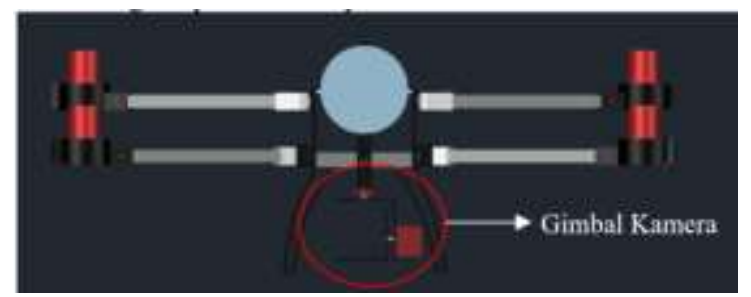

Figure 9. Design and Placement of Gimbal Camera

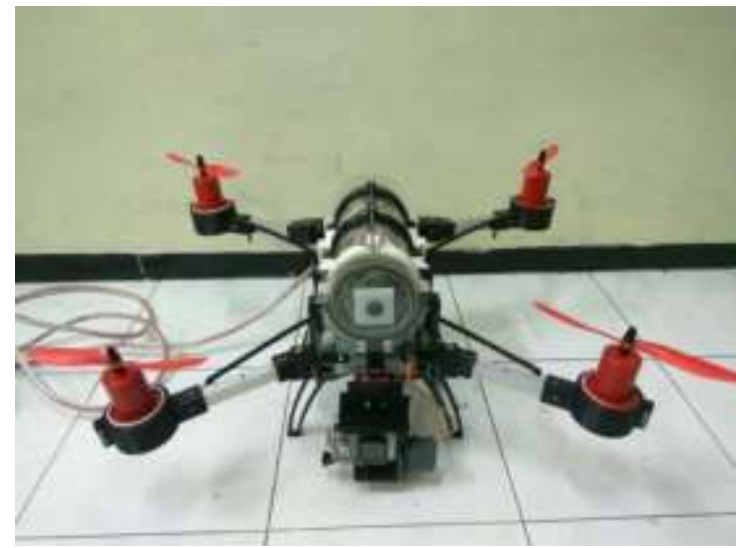

Figure 10. The Proposed Aquatic Quadcopter

\subsection{PID Control}

The method for maintaing the stability of ROV as well as camera gimbal is PID controller. The PID controller stabilizes pitch, roll, and yaw of ROV and also the depth. The proposed system uses MPU 6050 of IMU sensor for stabilize the position of ROV and MS5803 pressure sensor for maintaing the depth. It uses values which are taken from remote control to determine the setting point of roll, pitch, yaw, and depth.

\section{Experimental Results}

We have successfuly realized the proposed Aquatic Quadcopter as shown in figure 10. In order to measure to performance of the proposed system, we conducted several experiments such as performance of propulsion, performance of IMU sensor, performance of depth sensor, and performance of PID controller. The experiments were conducted in an aquarium with size is $90 \mathrm{Cm} \times 90 \mathrm{Cm} \times 100 \mathrm{Cm}$.

\subsection{Performance of Propulsions}

The experiment is aimed for measuring the performance of our realized propulsions. We measured the pull of the propulsion in the water by attaching the spring balance. It was noted that each propulsion has maximum pull of 1200 gram.

\subsection{Performance of IMU Sensor}

The experiment is aimed for measuring the accuracy of our IMU sensor to estimate the Aquatic Quadcopter position in roll and pitch direction. The experiment data are shown in figure 11 and figure 12 . The data show that the average accuracy error for roll and pitch are $1,37 \%$ and $1,53 \%$. 


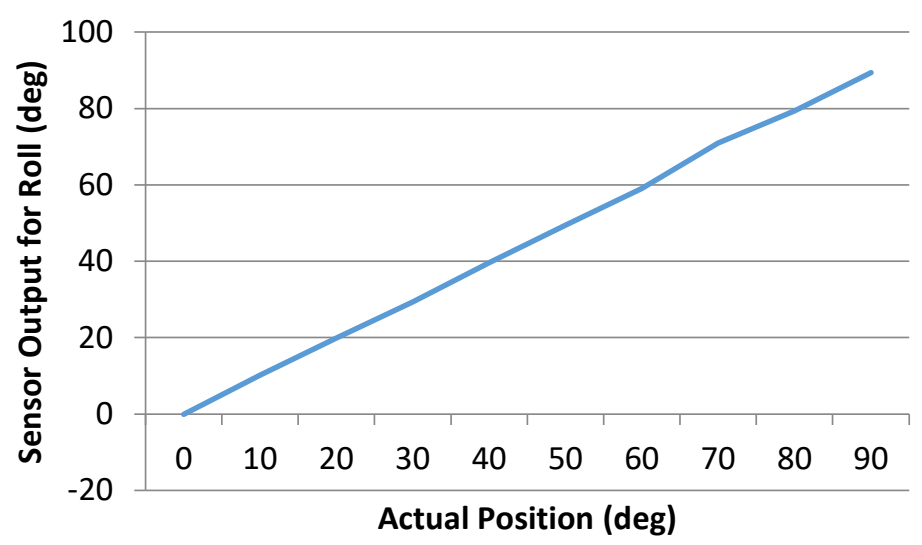

Figure 11. Accuracy of IMU Sensor to the Roll Position

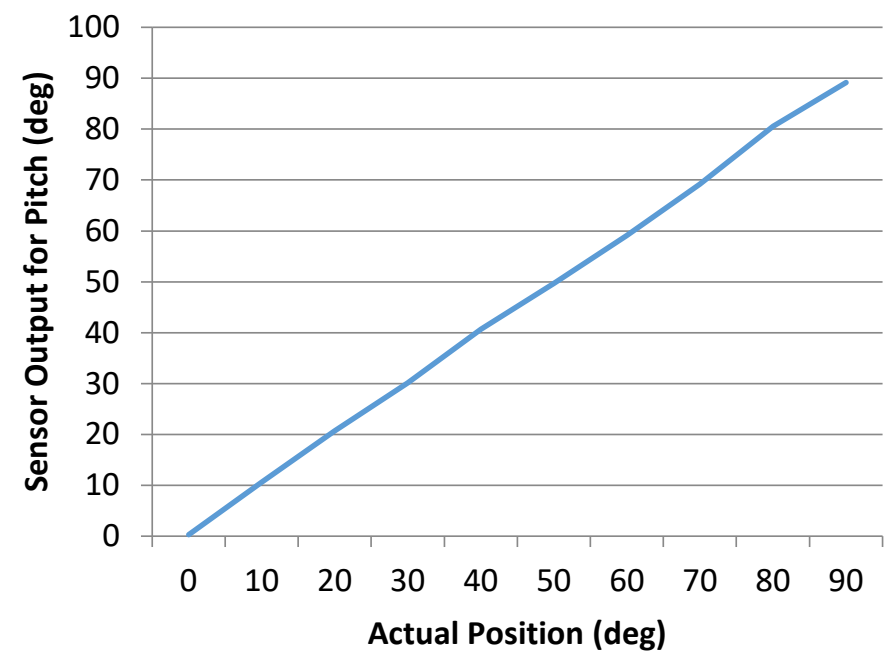

Figure 12. Accuracy of IMU Sensor to the Pitch Position

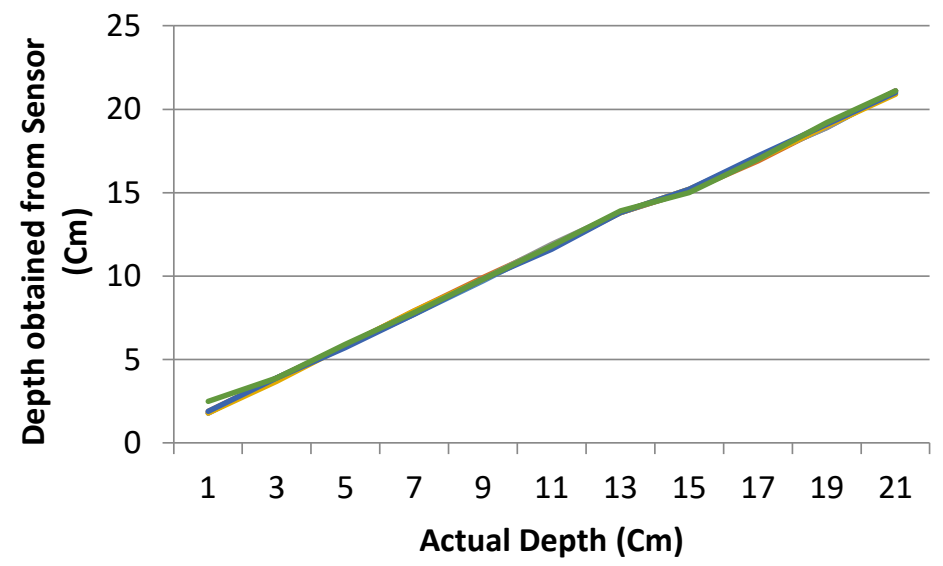

Figure 13. Accuracy of Depth Sensor 


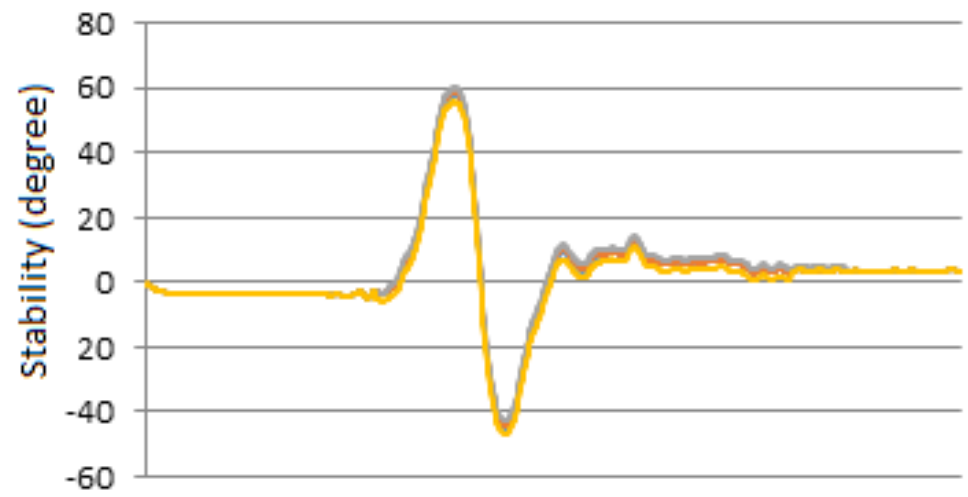

Figure 14. Stability

\subsection{Performance of Depth Sensor}

The experiment is aimed for measuring the accuracy of the depth sensor (MS 580314BA) while used to our application. The experiment was conducted by put in the sensor to aquarium, note the depth obtained from the sensor and compare it with the manual one. The result shows in figure 13. It shows that the average error is $1 \mathrm{Cm}$.

\subsection{Performance of PID Controller}

The experiment is aimed for measuring the performance of PID controller while controlling the roll, pitch, and depth position of aquatic quadcopter. The result of stability while disturbance taking into account shows in Figure 14.

\section{Conclusion}

The aquatic quadcopter with hold position and gimbal controller for capturing underwater video has been successfuly developed and realized. It can pull an object up to 1200 gram. It has the average accuracy error for roll and pitch are 1,37\% and 1,53\%. It has depth measurement accuracy is $1 \mathrm{Cm}$. The system also successfuly capture video smoothly due to it has been stabilized by using gimbal controller.

\section{References}

[1] A. Salman, "ROV: Robot Pintar di Bawah Laut", Jejak Hydrographer, (2007): s.n.

[2] N. Sakagami, F. Takemura, R. Ono, C. Katagiri, Y. Nakanishi and Y. Yamamoto, "Observation Support System of an ROV for Underwater Archaeology", International Conference on Intelligent Informatics and Biomedical Sciences (ICIIBMS), Okinawa, Japan, (2015).

[3] C. Joachim, R. Phadungthin and S. Srikitsuwan, "Design and Development of a Remotely Operated Underwater Vehicle", 2015. 16th International Conference on Research and Education in Mechatronics (REM), (2015), pp. 148 - 153.

[4] H. Alzu'bi, O. Akinsanya, N. Kaja, I. Mansour and O. Rawashdeh, "Evaluation of an Aerial Quadcopter Power-Plant for Underwater Operation", 10th International Symposium on Mechatronics and its Applications (ISMA), (2015), pp. 1-4.

[5] T. Inoue and J. Ishiwata, "Small-Size ROV Launched from Underwater TV System for Observation in Scientific Drillings", Oceans 2015, (2015), pp. 1-5.

[6] N. H. Tehrani, M. Heidari, Y. Zakeri and J. Ghaisari, "Development, Depth Control and Stability Analysis of an Underwater Remotely Operated Vehicle (ROV)", 8th IEEE International Conference on Control and Automation, Xiamen, China: s.n., (2010).

[7] A. Z. Abidin, R. Mardiyanto and D. Purwanto, "Implementation of PID Controller for Hold Altitude Control in Underwater Remotely Operated Vehicle", 2016 International Seminar on Intelligent Technology and Its Application (2016).

[8] A. Jafar, S. M. Ahmad and N. Ahmed, "Mathematical Modeling and Control Law Design for 1DOF Quadcopter Flight Dynamics", International Conference on Computing, Electronic and Electrical Engineering, (2016). 
[9] J. Y. Park, H. Shim, H. Baek, S. Yoo, B. H. Jun and P. M. Lee, "Multi-legged ROV Crabster and an acoustic camera for survey of underwater cultural heritages", Washington: s.n., (2015).

\section{Authors}

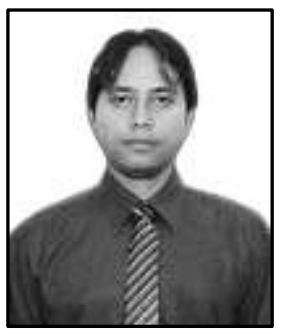

Ronny Mardiyanto

Address:

Kampus, Keputih, Sukolilo, Surabaya, Indonesia

Brief Biographical History:

2003 S.T. degree in Electric and Electronics Engineering, Institut Teknologi Sepuluh Nopember, Indonesia

2006 M.T. degree in Electric and Electronics Engineering, Institut Teknologi Bandung, Indonesia

2008- Ph.D. Student, Saga University, Japan

2003 - now. Lecturer, Institut Teknologi Sepuluh Nopember, Indonesia

Main Works:

- Robot Vision

- Electronics Instrumentation

- Unmanned Vehicle

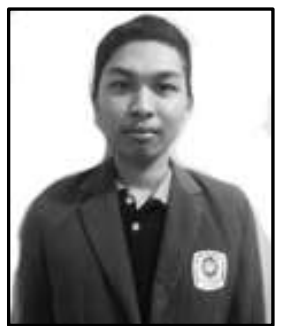

Komang Trisma Trisuta

\section{Address:}

Kampus, Keputih, Sukolilo, Surabaya, Indonesia

Brief Biographical History:

2017 S.T. degree in Electric and Electronics Engineering, Institut Teknologi Sepuluh Nopember, Indonesia

Main Works:

- Underwater Remotely Operated Robot 